\title{
Thermographic assessment of tensile response of metals subjected to varying levels of Plastic deformation
}

by T. Kathirvel*, V. Raghu Prakash* and B. Krishnan*

${ }^{*}$ Centre for Nondestructive Evaluation, Dept. of Mechanical Engineering, Indian Institute of Technology Madras, Chennai 600036 India \{me04d033, raghuprakash, balas\}@iitm.ac.in,

\begin{abstract}
Change in surface temperature of metallic materials due to residual plastic deformation is characterized by Infrared thermographic NDE technique. Residual plastic strain $\left(\varepsilon_{p}\right)$ was induced by loading a specimen to post-yield strain levels and by unloading thereafter to zero load. The observations from our experiments are, elastic limit is represented by an inflection point in temperature versus strain curve, which establishes a linear relationship with $\varepsilon_{\mathrm{p}}$. Hence it is possible to predict the extent of $\varepsilon_{p}$ in metallic materials using the inflection point measurements. The temperature from the elasto-plastic regime also has a linear dependence with the extent of residual plastic deformation in metallic materials.
\end{abstract}

\section{Introduction}

During service, materials experience different types of loading: static and dynamic. Depending on the nature and severity of loading, which at sometimes might be beyond the yield limit of a material, inducing residual plastic deformations, leading to accelerated damage and degradation of material. Characterizing material damage through appropriate measurement of mechanical properties under such situations becomes difficult esp. in case of in-service components. Direct measurement of damage by means of microscopic observations is not easy. Hence an indirect way is to deduce the damage from its influence on measurable properties such as variation in tensile properties (yield strength, ductility), stiffness loss, electrical resistance, ultrasonic velocity, ultrasonic attenuation, micro-hardness change, etc. NDE techniques such as nonlinear ultrasonics, acoustic emission, positron annihilation, Barkhausen noise emission and Infrared thermographic techniques are considered as candidate techniques for such property characterization. In this paper, Infrared thermographic technique was used for assessing the tensile response of metals subjected to various level of residual plastic strain.

\section{Experimental Setup}

Tensile dog-bone flat specimens conforming to ASTM E-8 standard with a gage length of $40 \mathrm{~mm}$, gage section width of $20 \mathrm{~mm}$ and gripping portion width of $25 \mathrm{~mm}$ was machined. Testing was carried out using a 100-kN servohydraulic Material Test System (MTS) under strain controlled tensile loading. Axial extensometer with a gage length of 25 $\mathrm{mm}$ and a travel of $+/-5 \mathrm{~mm}$ was used to estimate elongation in the gage section. Tensile tests were carried out at a strain rate of $0.75 \& 1.5 \mathrm{~mm} / \mathrm{min}$ in strain controlled mode for an incremental axial elongation of $0.5 / 0.75 \mathrm{~mm}$ over the extensometer gage length. The material is unloaded to zero force in force controlled mode. This loading and unloading pattern was repeated multiple times on each sample to introduce residual plastic strain and the surface temperature of the specimen was in-situ monitored using infrared camera.

The infrared camera used for the temperature measurements was a CEDIP Jade LWIR camera, cooled by electronic control of stirring cooler. The camera contains an $\mathrm{HgCdTe}$ detector which is sensitive to infrared radiation in the wavelength range 8-10 $\mu \mathrm{m}$ and equipped with $25 \mathrm{~mm}$-objective lens with a field of view of $22^{\circ} \times 16^{\circ}$. The camera was positioned on a tripod at a distance of approximately half a meter from the specimen surface. The image window size used in the present set of experiments is $76 \times 120$ pixels with integration time of $200 \mu$ s. Temporal resolution of the IR camera was $200 \mathrm{mK}$ and spatial resolution of IR images was $0.6 \mathrm{~mm}$. The temperature images were acquired in Automatic Gain Controlled (AGC) mode at a frame rate of 400 frames per second and the acquired data archived onto a computer. The temperature values were averaged over a circular area at the centre of the specimen gage length.

\section{Results and Discussion}

Temperature response for Mn-SS material subjected to tensile loading, unloading and subsequent reloading is shown in Fig. 1 and its stress-strain curve shown in Fig. 2. Temperature response due to tensile loading consists of two segments due to tensile elastic loading and tensile plastic loading. When the material is tensile loaded (stress increases) within elastic limit, there is a linear decrease in temperature (the first segment represented by ' $a$ ' in Fig. 1) due to thermoelastic effect. Towards the end of linear temperature variation, there is a non-linear drop in temperature, which is due to 
localized yielding. As the material is plastically deformed beyond yield point, material temperature increases (the second segment represented by 'b' in Fig. 1), as most of the mechanical work done on material is converted into heat. Similar temperature response was observed for the subsequent loadings. The point of inversion (i.e., the point of maximum temperature drop) in each loading is termed as inflection point. From the stress-strain curve, one could infer the following: yield strength and strain hardening coefficient increases as a function of residual plastic deformation and correspondingly, inflection point temperature and strain increases in temperature-strain curve (indicated as arrow (i) in Fig. 1). Temperature rise in elasto-plastic regime increases because of increase in number of heat sources (due to dislocation multiplication) indicated as arrow (ii) in Fig. 1.

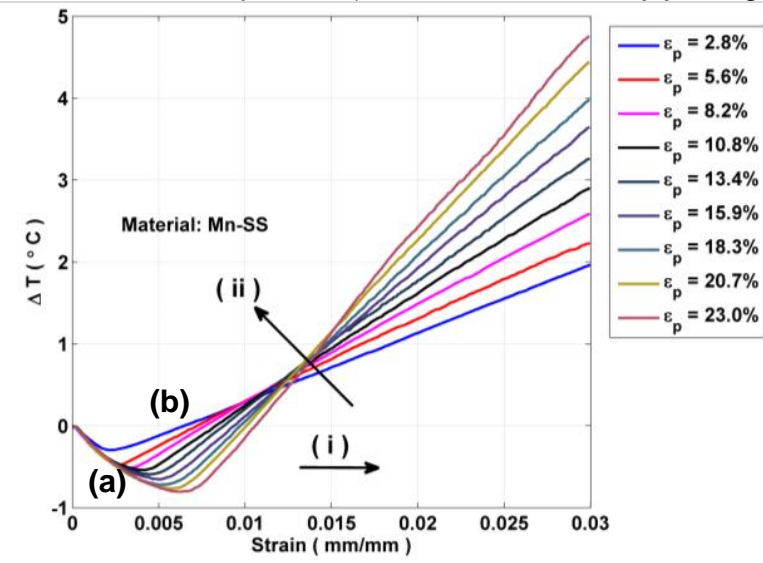

Fig. 1. Temperature-strain response during interrupted tensile test on Mn-SS material

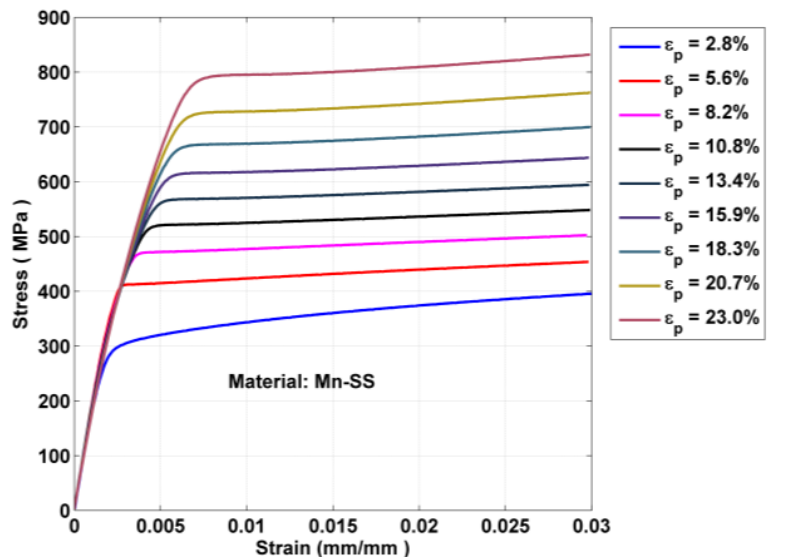

Fig. 2. Stress-strain response during interrupted tensile test on Mn-SS material

Figure 3 presents the variation of yield stress as a function of the inflection point strain for Mn-SS material tested at two different loading rates of $0.75 \mathrm{~mm} / \mathrm{min}$ and $1.5 \mathrm{~mm} / \mathrm{min}$. It was observed that there is a linear relationship between the yield point and the percentage of residual plastic deformation. At an inflection point strain of 0.002 (vertical arrow shown in Fig. 3), the stress value in the y-axis corresponds to the $0.2 \%$ yield stress of the material (horizontal arrow shown in Fig. 3) in the as-received condition.

Temperature rise during the tensile plastic loading of the specimen was observed to increase linearly as a function of applied strain. The slope of this temperature rise was referred to as thermo-plastic slope and the same was plotted versus the residual plastic strain induced in the material. Figure 4 presents the variation of thermoplastic slope vs. the residual plastic strain for Mn-SS material at two different rates of loading. A linear fit that describes the variation is also shown in the same figure. Thus, on the basis of the thermo-plastic slope, which is unique for a given loading rate for a material, it is possible to estimate the extent of prior residual plastic strain induced in the material.

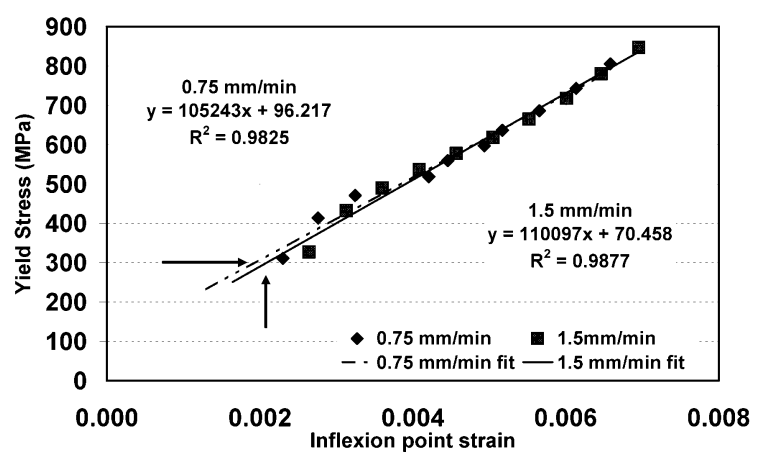

Fig. 3. Yield stress vs. inflection point strain response during interrupted tensile test on Mn-SS material

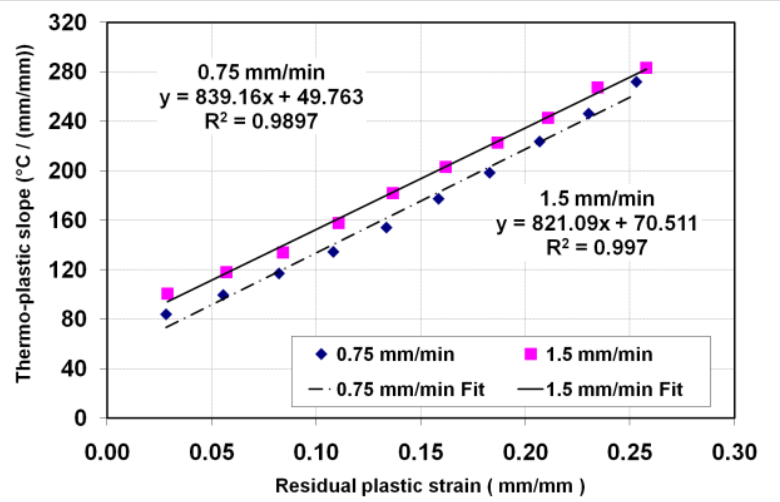

Fig. 4. Thermo-plastic slope vs. residual plastic strain response during interrupted tensile test on Mn-SS

\section{Conclusions}

Infrared thermographic technique can be employed for the tensile characterization of metallic materials subjected to various levels of residual plastic deformation. The inflection point is prominent for the materials considered and there is a sufficient correlation between the inflection point and the elastic limit. There is a linear correlation between the strain at the inflection point and $0.2 \%$ proof stress determined from stress-strain curve. The thermo-plastic slope from temperature-strain curve varies linearly with the residual plastic strain. 